\title{
A PURE GEOMETRIC APPROACH TO COSMOLOGY
}

\author{
M.I.WANAS \\ Astronomy Department, Faculty of Science \\ Cairo University, Giza, Egypt e.mail wanas@frcu.eun.eg
}

It is well known that standard Big-Bang cosmology suffers from certain problems, e.g. singularity, horizon, flatness, ... In the present work it is claimed that the appearence of some of these problems is due to two main assumptions. The first is the assumption that the 4-dimentional Riemannian (RIE)-geometry gives a complete description of the cosmic space-time. The second is the assumption that the material distribution in the universe is described by a phenomenological (PH)-matter tensor. It is shown that, by relaxing these two assumptions, some of the problems of the standard BigBang cosmology could be avoided. The following table summarises some results in favour of the above claim. The absolute parallelism (AP)-geometry is used to construct some of the theories mentioned in the table.

TABLE 1. Comparison between isotropic and homogeneous models

\begin{tabular}{llllll}
\hline Field theory & Geometry & Matter tensor & Horizon? & Flatness? & Reference \\
\hline GRT & RIE & PH & Yes & Yes & 1 \\
GFT & AP & Geometric & No & No & 2 \\
MTT & AP & PH & Yes & Yes & 3 \\
NGR & AP & PH & Conditional & Yes & 4 \\
\hline
\end{tabular}

\section{References}

1.Einstein, A. (1955) The Meaning of Relativity, Princeton.

2.Wanas, M.I. (1989) Astrophys. Space Sci., 154, 165.

3.Saez, D. And De Juan (1984) Gen. Rel. Grav., 16, 5.

4.Mikhail, F.I., Wanas,M.I. and Nashed, G.G.L. (1995) Astrophys. Space Sci., 228, 225. 